\section{Sorting out sortilin}

\section{By Tim Fulmer, Senior Writer}

An international team of cardiovascular researchers has reported the largest ever genomewide association study meta-analysis, encompassing 46 data sets and leading to the identification of 59 new genetic variants associated with plasma lipid levels. ${ }^{1}$ Potentially the most provocative finding is an association between coronary artery disease and a mutation in the gene for sortilin 1, an intracellular trafficking protein involved in hepatic lipid metabolism. ${ }^{2}$

The team is now studying the sortilin 1 (SORT1) pathway in animals and has partnered with RNAi company Alnylam Pharmaceuticals Inc., which has exclusive rights to develop and commercialize any SORT1-targeting therapeutic that results from the research.

Previous GWA studies enrolling up to 20,000 individuals of European ancestry had identified SNP variants at 36 genetic loci that contributed to variation in plasma lipid levels. Those studies provided insights into the genetic variation underlying lipid metabolism and dyslipidemia but did not clarify how those loci might also contribute to cardiovascular conditions like coronary artery disease (CAD) and myocardial infarction (MI).

Thus, a group led by Sekar Kathiresan and Daniel Rader carried out a meta-analysis of 46 previously reported GWA studies to identify additional lipid-associated variants and determine whether any of those variants significantly contributed to cardiovascular disease.

Kathiresan is director of preventive cardiology at Massachusetts General Hospital and assistant professor of medicine at Harvard Medical School. Rader is director of the Preventive Cardiovascular Medicine and Lipid Clinic and professor of medicine at the University of Pennsylvania School of Medicine.

The meta-analysis of more than 100,000 individuals of European ancestry looked for associations between about 2.6 million SNPs and 4 heritable lipid traits: total cholesterol, low-density lipoprotein (LDL) cholesterol, high-density lipoprotein (HDL) cholesterol and triglycerides.

With a threshold of statistical significance set at $p<5 \times 10^{-8}$, which is standard for GWA studies, the analysis identified SNPs in 95 lociincluding 59 new ones-that were significantly associated with at least 1 of the 4 risk factors.

In additional cohorts of East Asians, South Asians and African Americans, a majority of the 95 loci showed the same level of association with the 4 risk factors that was seen in the European cohort. Thus, the researchers concluded in a paper in Nature that most of the loci "contribute to the genetic architecture of lipid traits widely across global populations."

The next question was how strongly the loci are associated with risk for CAD. To find out, the researchers genotyped 24,607 CAD patients of European descent and 66,197 non-CAD controls. Of the 95 lipid-related loci, 14 were significantly associated with CAD $(p<0.001)$.

In an accompanying paper in Nature, the same researchers provided an example of how the GWA study findings might be used to guide the identification of new therapeutic targets.

Here, the team zeroed in on loci on chromosome 1p13. Of the new SNPs identified in the meta-analysis, those at the $1 \mathrm{p} 13$ loci were most strongly associated with LDL cholesterol, a marker of cardiovascular disease risk. Independent work by other researchers has shown an association between SNPs on 1p13 and MI in humans. ${ }^{3,4}$

Fine-mapping studies then led to the identification of a single SNP in a noncoding region of DNA that caused decreases in hepatic expression of SORT1, a Golgi protein that had not previously been associated with lipid or cardiovascular disorders.

The final question was whether low SORT1 expression caused the high plasma LDL levels that are associated with risk for MI and CAD.

To find the answer, the researchers used small interfering RNA knockdown to decrease Sort 1 expression or adeno-associated viral (AAV) vectors to increase Sortl activity in mice. The data showed a causal relation: high and low expression of Sort1 in the liver led to low and high plasma LDL, respectively.

The group concluded that the SORT1 pathway is "a promising new target for therapeutic intervention in the reduction of LDL-C and prevention of MI." 2

The team on the paper describing the SORT1 research included researchers from Alnylam. According to Kevin Fitzgerald, the biotech's senior director of research, Alnylam holds rights to develop and commercialize any RNAi therapeutics that derive from the research collaboration between the company and the labs of Kathiresan and Rader.

In a commentary accompanying the two papers, Alan Shuldiner and Toni Pollin wrote that the SORT1 study is an "example of how information from GWAS can be used to unravel new regulatory pathways that alter the risk of human disease, in this case myocardial infarction." ${ }^{5}$

Shuldiner is professor of medicine and Pollin is assistant professor of medicine at the University of Maryland School of Medicine's Division of Endocrinology, Diabetes and Nutrition.

\section{Sorting through SNPs}

Although the findings imply that increasing SORT1 expression in the liver could reduce plasma LDL and thus lower the risk of cardiovascular disease, "more cell biology studies and mechanistic work is needed to determine whether the optimal way of doing that is targeting sortilin 


\section{ANALYSIS}

\section{TARGETS \& MECHANISMS}

directly or targeting a regulator of sortilin activity," co-corresponding author Kathiresan told SciBX.

Going forward, the Kathiresan and Rader groups will continue to use a combination of GWA studies and animal work to sort through the 95 loci to home in on additional genes that drive variation in plasma lipid levels and contribute to cardiovascular disease.

"As we move forward, we'll initially focus on the 14 loci in the metaanalysis that are associated with both lipid levels and diseases such as CAD and MI," said Kathiresan. "That ongoing work includes studying the role of the sortilin pathway in lipid metabolism and cardiovascular disease and developing strategies for targeting that pathway."

Co-corresponding author Rader told SciBX the studies are looking at ways of modulating SORT1 expression in mice as well as in cultured human hepatocytes.

Kathiresan and Rader are collaborating with Alnylam "to mechanistically evaluate the metabolic and molecular effects of novel genes implicated by human genetic studies in cardiovascular disease and to develop RNAi therapeutics against those gene candidates," said Alnylam's Fitzgerald.

RNAi helps link complex genetic loci to specific pathways and mechanisms and, ultimately, to individual target genes, he added.

In parallel with studies to identify new therapeutic targets, Kathiresan told SciBX he wants to explore using the 95 loci or a subset of them as a diagnostic to guide treatment of cardiovascular disorders.

"Given the potential link between those 95 loci and risk for lipid disorders and cardiovascular disease, one could hypothesize the use of a risk score based on those loci that would inform, for example, when to put patients on early statins to help lower their risk for developing coronary artery disease and MI," Kathiresan said. Though it remains to be seen, a panel of risk loci could offer a more sensitive diagnostic for when to begin statin therapy than a single marker like plasma cholesterol, he added.

According to Kathiresan, the 95 loci and SNPs identified in the two papers, including SORT1, are in the public domain.

Fulmer, T. SciBX 3(32); doi:10.1038/scibx.2010.973

Published online Aug. 19, 2010

\section{REFERENCES}

1. Teslovich, T.M. et al. Nature; published online Aug. 5, 2010; doi:10.1038/nature09270

Contact: Sekar Kathiresan, Massachusetts General Hospital, Boston, Mass.

e-mail: skathiresan@partners.org

2. Musunuru, K. et al. Nature; published online Aug. 5, 2010; doi:10.1038/nature09266

Contact: Daniel Rader, University of Pennsylvania School of Medicine, Philadelphia, Pa.

e-mail: rader@mail.med.upeen.edu

Contact: Sekar Kathiresan, Massachusetts General Hospital, Boston, Mass.

e-mail: skathiresan@partners.org

3. Samani, N.J. et al. N. Engl. J. Med. 357, 443-452 (2007)

4. Myocardial Infarction Genetics Consortium. Nat. Genet. 41, 334-341 (2009)

5. Shuldiner, A.R. \& Pollin, T.I. Nature 466, 703-704 (2010)

COMPANIES AND INSTITUTIONS MENTIONED

Alnylam Pharmaceuticals Inc. (NASDAQ:ALNY), Cambridge, Mass.

Harvard Medical School, Boston, Mass.

Massachusetts General Hospital, Boston, Mass.

University of Maryland School of Medicine, Baltimore, Md.

University of Pennsylvania School of Medicine, Philadelphia, Pa. 